\title{
LYMPHOMES MALINS NON-HODGKINIENS PRIMITIFS DES AMYGDALES PALATINES
}

\author{
I. HARIGA, S. ZRIBI, A. BOUZAYANI, F. BEL HADJ YOUNES, K. KHAMMASSI, M. BEN AMOR, \\ O. BEN GAMRA, CH. M'BAREK, A. EL KHEDIM
}

SERVICE D'ORL ET DE CHIRURGIE CERVICO-FACIALE. L'HÔPITAL HABIB THAMEUR.TUNIS

\begin{abstract}
RESUME
Introduction : les amygdales palatines constituent la première localisation extra ganglionnaire des lymphomes malins non hodgkiniens cervico-faciaux. II s'agit d'un lymphome de type B dans la majorité des cas.

Matériel et méthodes : Nous avons colligé 3 cas de LMNH primitifs de l'amygdale palatine, entre 1995 et 2007

Résultats : II s'agit de trois hommes âgés respectivement de 15, 42 et 72 ans qui ont consulté pour une odynophagie persistante évoluant depuis 3 mois en moyenne. L'examen a objective, dans tous les cas, une hypertrophie amygdalienne unilatérale à surface ulcérée. Tous nos patients ont eu une amygdalectomie à visée diagnostique. L'examen anatomopathologique a conclu à un lymphome malin non Hodgkinien de phénotype B, chez nos trois malades. Le bilan d'extension était négatif. Le traitement a consisté en une chimiothérapie exclusive pour deux patients et une radio chimiothérapie pour le troisième. L'évolution était favorable pour les deux jeunes patients avec un recul de 3 ans. Le troisième patient est décédé d'une maladie intercurrente.

Conclusion : Les lymphomes malins non-Hodgkiniens primitifs de l'amygdale palatine sont rares. Le diagnostic précoce est souvent difficile. La décision thérapeutique ne se discute qu'après un diagnostic histologique clairement établi, suivi d'un bilan de la maladie et du malade. Leur pronostic dépend du stade de la maladie.

Mots-clés : Lymphome non hodgkinien, Cavité buccale, Radiothérapie, Chimiothérapie, Anticancéreux, Hémopathie maligne, Cavité buccale pathologie.
\end{abstract}

\section{SUMMARY}

Introduction: The most predominant localization for extranodal non-hodgkinien lymphoma (NHL) in the head and neck region is the tonsil. The vast majority of $\mathrm{NHL}$ at this site is $\mathrm{B}$-cell lymphomas.

Materials and methods: The authors presented three cases of primary Non Hodgkin's Lymphoma of the tonsil, treated between 1995 and 2007

Results: we report the cases of three men aged respectively 15, 42 and 72 years. They complained of a persistent odynophagia during three months ago. Clinical examination detected unilateral enlarged tonsil with ulcerated surface. All of them have bilateral tonsillectomy. The histopathologic examination concluded at a NHL with a B phenotype.

The treatment consisted on chemotherapy for two patients and on radio and chemotherapy for the third patient. We have noted one death for our three patients.

Conclusion: Primary NHL of the tonsil is rare. An advanced diagnosis is often difficult. Both histopathologic identification of the tumor and evaluation of the patient are essentiel for the therapeutic decision. Prognostic depends on the stage of the lymphoma.

Keywords: Non Hodgkin lymphoma, Oral cavity, Radiotherapy, Chemotherapy, antineoplastic agent, malignant hemopathy, Oral cavity disease.

\section{INTRODUCTION}

Les lymphomes malins non Hodgkiniens (LMNH) sont des proliférations malignes du tissu lymphoïde. Ils représentent $5 \%$ des tumeurs malignes de la tête et du cou et se développent préférentiellement à partir du tissu lymphoïde extra ganglionnaire $(1,2)$. La localisation au niveau de l'anneau de Waldeyer représente environ 10\% de tous les LMNH et plus du tiers des localisations extra ganglionnaires.

Les LMNH se présentent sous la forme de différentes entités reconnues au sein de nouvelles classifications qui intègrent les données de l'immunophénotypage moderne (4). Le type histologique est plus déterminant dans la prise en charge thérapeutique que la localisation anato- mique. De ce fait, la décision ne se discute qu'après un diagnostic histologique clairement établi. Cette prise en charge est multidisciplinaire et repose sur la radio-chimiothérapie.

\section{MATERIEL ET METHODES :}

C'est une étude rétrospective à propos de 3 dossiers de patients atteints de lymphomes non-Hodgkiniens primitifs de l'amygdale palatine recueillis sur une période de 11 ans entre 1997 et 2007. A travers ces observations, nous rappelons les données épidémiologiques, cliniques, paracliniques, thérapeutiques et pronostiques de cette pathologie. 


\section{OBSERVATIONS}

\section{Observation 1}

II s'agit d'un homme âgé de 72 ans, tabagique à 20 PA, atteint d'une cécité bilatérale depuis un an non explorée, qui s'est présenté pour une dysphagie haute associée à une odynophagie évoluant depuis 3 mois, dans un contexte d'altération de l'état général.

L'examen clinique a montré une amygdale palatine gauche hypertrophiée $(4 \mathrm{~cm})$ à muqueuse ulcérée, avec une polyadénopathie cervicale homolatérale, ferme, mobile et indolore. L'endoscopie nasale était normale et la biopsie du cavum réalisée était normale.

La numération formule sanguine était normale et la vitesse de sédimentation était accélérée à $30 \mathrm{~mm}$ à la première heure. L'examen anatomopathologique de la pièce d'amygdalectomie a mis en évidence un lymphome amygdalien diffus à grandes cellules de phénotype $\mathrm{B}$. Le bilan d'extension comportant une radiographie du thorax, et un scanner cervico thoraco abdominal était négatif. II s'agissait d'une forme primitive localisée à l'amygdale palatine (stade I).

Le patient a eu une cure de chimiothérapie protocole CHOP (Adréblastine, Endoxan, Oncovin, Prednisone) avec une bonne évolution. II est décédé d'une maladie intercurrente 7 mois aprés.

Observation 2

II s'agit d'un garçon âgé de 15 ans, sans antécédents pathologiques particuliers, qui a consulté pour une odynophagie évoluant progressivement depuis 4 mois sans dysphagie ni signes généraux.

L'examen clinique a objectivé une amygdale palatine gauche hypertrophiée, congestive, irrégulière et à centre blanchâtre ulcéré. Les aires ganglionnaires cervicales et extra cervicales étaient libres. II n'y avait pas d'hépato splénomégalie. L'endoscopie nasale a révélé un cavum libre.

L'examen anatomopathologique de la pièce d'amygdalectomie a mis en évidence un lymphome malin non hodgkinien à grandes cellules $\mathrm{B}$. La biologie a montré une hyperleucocytose à $11200 / \mathrm{mm} 3$ et une vitesse de sédimentation à $12 \mathrm{~mm}$ à la première heure. Le taux de LDH était normal. Une biopsie médullaire était normale. La radiographie du thorax ainsi que le scanner cervico thoraco-abdominal étaient sans particularités. Le bilan d'extension de la maladie était donc négatif. II s'agissait alors d'un LMNH classé stade I de Ann Arbor.

Le patient a reçu six cures de chimiothérapie : protocole CHOP (Adréblastine, Endoxan, Oncovin, Prednisone). L'évolution était favorable avec une rémission complète avec un recul de 3 ans.

\section{Observation 3}

II s'agissait d'un homme âgé de 42 ans, suivi pour une maladie de Behçet et qui se plaint d'une odynophagie évoluant depuis 3 mois rebelle au traitement symptomatique associée à une tuméfaction latéro-cervicale haute droite évoluant dans un contexte d'altération de l'état général.

L'examen a révèlé une lésion ulcéro-bourgeonnante de l'amygdale palatine droite avec une adénopathie jugulocarotidienne homolatérale de $4 \mathrm{~cm}$ de grand axe (figure 1).

La TDM a objectivé une tumeur de l'amygdale droite infiltrant la base de la langue et les espaces parapharyngés droits (figures 2 et 3 ). L'examen anatomopathologique de la pièce d'amygdalectomie a conclu à un lymphome à grandes cellules B. Sur le plan biologique, le taux de LDH était élevé (supérieur à $3 \mathrm{mg} / \mathrm{l}$ ). La numération formule sanguine ainsi que la vitesse de sédimentation était sans anomalies. Dans le cadre du bilan d'extension, la fibroscopie digestive ainsi que la biopsie médullaire étaient normales. II s'agissait alors d'un LMNH stade II de Ann Arbor avec une localisation amygdalienne et une localisation ganglionnaire cervicale homolatérale.

Un traitement comprenant quatre cures de chimiothérapie type ACVBP (Adréblastine, Endoxan, Oncovin, Bléomycine, Prednisone) et une radiothérapie externe ont été entrepris. L'évolution était favorable avec un recul de 3 ans.

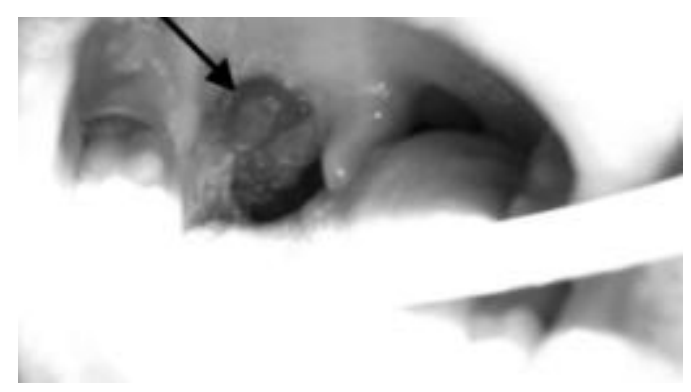

Fig. 1: Examen oropharyngé : hypertrophie unilatérale de l'amygdale palatine droite à surface ulcérée.

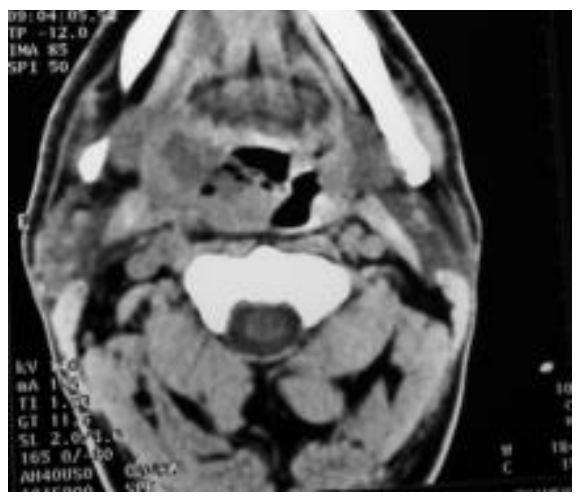

Fig. 2 : Scanner cervical en coupe axiale : tumeur de l'amygdale palatine droite infiltrant la base de langue et les espaces parapharyngés droits. 


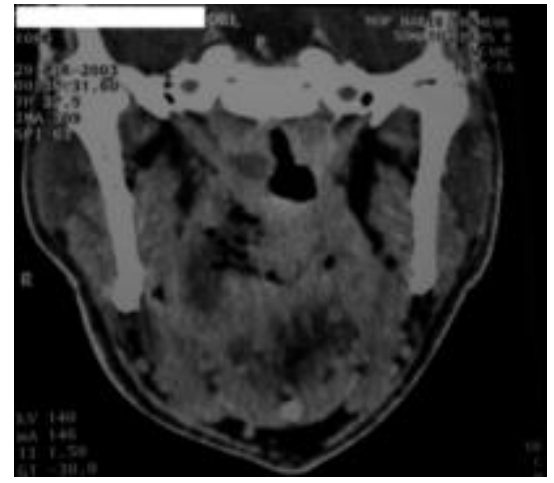

Fig. 3 : Scanner cervical en coupe axiale :

tumeur de l'amygdale palatine droite infiltrant la base de langue et les espaces parapharyngés droits.

\section{DISCUSSION}

La majorité des tumeurs malignes de l'amygdale palatine sont des carcinomes. Les LMNH primitifs des amygdales palatines occupent la 2ième place avec une fréquence de 5 à $14 \%(3,5)$. Parmi les LMNH de l'anneau de Waldeyer, les amygdales palatines représentent le premier site (40 à $79 \%$ des lésions primitives), suivi par le nasopharynx (25 à 35\%) et l'amygdale linguale (3 à 10\%) (3). L'atteinte multiple se voit dans près de $5 \%$ des cas de lymphome amygdalien. Cette atteinte est bilatérale dans 10\% des cas (4). Les épithéliomes, les plasmocytomes, les adénocarcinomes, les mélanomes, la maladie de Hodgkin, les sarcomes et les métastases constituent des localisations tumorales rares de l'amygdale palatine. Un risque accru de survenue d'un LMNH a été constaté chez les patients porteurs de maladies auto-immunes, particulièrement, le syndrome sec qui multiplie ce risque par 40 (6). En effet notre troisième cas est connu porteur d'une maladie de Behçet. D'autres facteurs favorisent la survenue de LMNH et expliquent l'accroissement actuel de l'incidence de cette maladie, tels que: le SIDA où le lymphome $B$ constitue un néoplasme opportuniste, les déficits immunitaires acquis après transplantation d'organe avec traitement immunosuppresseur $(6,7)$.

Les LMNH s'observent à tout âge, mais l'âge moyen de survenue se situe vers 59 ans. Plus de $80 \%$ des LMNH de l'anneau de Waldeyer surviennent après l'âge de 50 ans $(7,8,9)$. Dans notre étude, l'âge moyen est de 43 ans (15 ans-72 ans). La plupart des séries de la littérature décrivent une distribution égale de la maladie selon le sexe. Parfois, une légère prédominance masculine est retrouvée (10). Les trois patients de notre série sont de sexe masculin.

Les LMNH de l'amygdale s'installent rapidement avec des signes d'appel non spécifiques, à type d'asymétrie amygdalienne, d'odynophagie, de dysphagie haute et de tuméfaction cervicale, tel est le cas des malades de notre série. Ces signes peuvent prêter confusion avec une simple angine. Le diagnostic est alors souvent méconnu et tardif (11). C'est la persistance de la symptomatologie malgré un traitement bien conduit qui fait évoquer le diagnostic. L'atteinte amygdalienne bilatérale est rare et représente $10 \%$ des cas.

Les signes systémiques, peu communs et présents uniquement dans $25 \%$ des cas, sont d'une aide au diagnostic $(7,12)$. L'atteinte ganglionnaire associée est fréquente et s'observe dans $2 / 3$ des cas environ. Elle est souvent homolatérale à la lésion. Ces adénopathies sont volumineuses mais restent fermes, mobiles et indolores, bien différentes des métastases ganglionnaires des carcinomes épidermoïdes (4). Au moment du diagnostic, l'importance du volume de la tumeur est frappante. Elle est ferme sans être dure, évoluant sous une muqueuse non ulcérée, contrastant avec l'absence de douleur. Ce tableau peut faire évoquer un phlegmon péri-amygdalien, mais l'absence de signes infectieux, de douleur, de trismus et d'œdème de la luette doit alerter l'examinateur. Malheureusement, il n'est pas exceptionnel de voir ces lésions incisées à tort en première intention (4).

L'imagerie n'est pas contributive au diagnostic positif d'autant plus qu'une petite asymétrie de taille entre les deux tonsilles est considérée comme normale. Cependant, l'imagerie peut être intéressante dans l'appréciation du volume tumoral et dans la détection des adénopathies non palpables notamment rétropharyngées. L'absence d'invasion des structures adjacentes de l'amygdale malgré l'importance de la masse tumorale est en faveur d'une localisation lymphomateuse (12).

Devant une suspicion clinique de lymphome de l'amygdale, seule la biopsie permet de confirmer le diagnostic. L'examen histologique comporte: une analyse morphologique simple de l'architecture du tissu prélevé (envahissement tumoral diffus ou organisé en follicules), une Immuno-Histo-Chimie, qui confirme le caractère lymphoïde de la masse tumorale et établit son phénotype $\mathrm{B}$ ou $\mathrm{T}$ et recherche d'autres marqueurs propres à chaque soustype et enfin, une analyse cytogénétique (à la recherche d'anomalies chromosomiques). L'étude en biologie moléculaire permet la détection de transcrits issus de translocation ou la mise en évidence d'une clonalité. (4)

L'interrogatoire recherche des signes généraux (amaigrissement supérieur à $10 \%$, sueurs nocturnes, fièvre supérieure à $38^{\circ} \mathrm{C}$ ), l'index d'activité ou performans status (PS) et des facteurs de risque (immunodéficience congénitale ou acquise, maladie auto-immune...).

L'examen général, doit explorer toutes les aires ganglionnaires et rechercher une éventuelle hépato-splénomégalie. Le bilan d'extension tumorale comporte d'une façon systématique une biopsie médullaire, une radiographie du thorax et souvent un scanner thoraco-abdomino-pelvien à la recherche d'adénopathies profondes.

Une endoscopie des voies aéro-digestives supérieures et une fibroscopie oeso-gastro-duodénale sont également demandées d'une façon systématique. L'atteinte digesti- 
ve associée à celle amygdalienne peut atteindre les $20 \%$, selon Hanna et al (13). Dans notre série, aucune localisation digestive n'a été trouvée. Un examen cytologique du LCR est systématiquement fait chez les patients porteurs d'un lymphome agressif. La ponction lombaire n'a été pratiquée pour aucun de nos patients.

Sur le plan biologique, l'hémogramme recherche des signes d'insuffisance médullaire. Le dosage de la lacticodéshydrogénase ( $\mathrm{LDH}$ ) et de la $\beta 2$ microglobuline, toutes deux corrélées à la masse tumorale, sont effectuées de principe pour leur valeur pronostique (7). Dans notre série, un taux élevé de LDH n'a été noté que chez le troisième malade.

Cette étape du bilan permet de classer la maladie selon les stades d'Ann Arbor:

- Stade I : avec atteinte seulement du site ayant conduit au diagnostic. C'est le cas de nos deux premiers patients - Stade II : s'ils s'associent des adénopathies périphériques du même côté du diaphragme. c'est le cas du troisième patient.

- Stade III : atteinte ganglionnaire sus et sous-diaphragmatique.

- Stade IV : atteinte unique extra-ganglionnaire avec atteinte ganglionnaire non contiguë ou plusieurs atteintes extra-ganglionnaires.

Le bilan du malade inclut la fonction myocardique et, outre la biologie standard, les sérologies VIH, HTLV-1, $E B V$ et hépatite $C$ en vue de rechercher un contexte viral ayant pu favoriser la survenue du lymphome. Enfin une évaluation stomatologique doit se faire en vue d'une irradiation.

Au terme de ce bilan, on peut identifier un certain nombre d'éléments pronostiques péjoratifs $(14,15)$ :

- l'âge supérieur à 60 ans.

- le siège de l'atteinte.

- le volume de la plus grosse masse supérieur à $5 \mathrm{~cm} 3$.

- le stade III ou IV.

- l'envahissement médullaire ou des localisations neurologiques.

- le taux élevé de LDH ou un taux de $\beta 2$ microglobuline supérieur à $3 \mathrm{mg} / \mathrm{l}$.

- les lymphomes de la lignée $T$, les lymphomes diffus à grandes cellules anaplasiques, les lymphomes lymphoblastiques et les lymphomes de Burkitt.

- les anomalies des chromosomes 6, 7 et 17 .

- L'infection par le VIH.

- l'index d'activité ou performans status (PS)

L'index pronostique international (IPI), qui prend en compte cinq de ces facteurs (âge supérieur à 60 ans), PS supérieur à 2 , stade III ou IV, taux élevé de LDH, présence de plus d'une localisation extra-ganglionnaire), permet de séparer les patients au profil évolutif différent et d'adapter la thérapeutique des lymphomes agressifs (7).
Les LNH de l'amygdale palatine sont presque toujours de phénotype $B$, avec une prédominance des formes diffuses à grandes cellules sur les lymphomes folliculaires et du manteau. II existe de rares cas de lymphomes anaplasiques $T$ (16). Tous nos cas sont des LMNH à grandes cellules de phénotype $B$.

Ces lymphomes diffus à grandes cellules sont des tumeurs agressives, caractérisées par une évolution spontanée rapide, et de ce fait, la présentation initiale est localisée (stades I et II) dans un peu plus du tiers des cas $(4,12)$.

Le traitement de première ligne est adapté aux facteurs pronostiques initiaux décrits précédemment. II repose sur la chimiothérapie qui seule peut donner une chance de guérison. La radiothérapie ne peut jouer qu'un rôle adjuvant (4). D'après Fisher et al (17), la chimiothérapie de référence est le protocole $\mathrm{CHOP}$ (cyclophosphamide, doxorubicine, vincristine, prednisone) qui est mieux toléré que les protocoles de troisième génération. Ce protocole a été livré à notre deuxième malade avec obtention d'une rémission complète avec un recul de 3 ans. Pour certains auteurs, une radiothérapie exclusive semble être un traitement adéquat pour les lymphomes stades I et II de bas grade (18). L'association à la chimiothérapie d'une irradiation des territoires ganglionnaires atteints semble améliorer significativement le pronostic. C'est le cas de notre troisième malade avec obtention d'une rémission complète après un recul de 3 ans (19).

La survie à 5 ans pour les LNH confinés à l'amygdale palatine est de $86 \%$. Ce chiffre diminue à $41 \%$ en présence d'adénopathie (8). Le seul cas de décès dans notre série est dû à une maladie intercurrente. Les rechutes sont fréquentes et indiquent un mauvais pronostic. Elles surviennent surtout dans les deux premières années après le traitement. Elles siègent à distance du site primitif, particulièrement dans les territoires ganglionnaires non irradiés et dans le tractus gastro-intestinal (13). Aucun cas de récidive n'a été noté dans notre série.

\section{CONCLUSION}

Les lymphomes malins non-Hodgkiniens primitifs de l'amygdale palatine sont rares. Leur diagnostic est évoqué devant une hypertrophie amygdalienne unilatérale et confirmé par la biopsie. Le diagnostic précoce est difficile (stade nodulaire intra-parenchymateux). La décision thérapeutique ne se discute qu'après un diagnostic histologique clairement établi, suivi d'un bilan de la maladie et du malade. Le pronostic dépend du stade. L'index pronostique international (IPI) permet de séparer les patients au profil évolutif différent et d'adapter la thérapeutique des lymphomes agressifs. 


\section{REFERENCES}

1. Endo S., Kida A., Sawada U. et al. Clinical analysis of malignant lymphomas of tonsils. Acta Otolaryngol (Stockh) 1996; (523): 263-6.

2. Saul S.H., Kapadia S.B. Primary lymphoma of Waldeyer's ring. Clinicopathologic study of 68 cases. Cancer 1985; 56:157-66.

3. Collins S., Spector G.J. Cancer of the oral cavity, oropharynx and pharynx. In: Ballenger JJ editor. Diseases of the nose, throat, ear, head and neck (13th edn). Philadelphia, PA: ea \& Febiger. 1985: 659-64.

4. Musck J.N., Schwaab G. Lymphomes des voies aérodigestives supérieures. Encycl Méd Chir. Oto-Rhino-Laryngologie. 2001; 20: 957-963

5. De Pena C.A., Van Tassel P., Lee Y.Y. Lymphoma of the head and neck. Radiol Clin North Am 1990; 28:723-43.

6. Urquhart A., Berg R. Hodgkin's and non-Hodgkin's lymphoma of the head and neck. Laryngoscope. 2001; 111:1565-1569.

7. Brette M., Monteil J.P. Manifestations oto-rhino-laryngologiques des hémopathies de l'adulte. EMC Oto-Rhino-Laryngologie. 2004; 56-72.

8. Barton J.H., Osborne B.M., Utler J.J. et al. Non'Hodgkin's lymphoma of the tonsil. A clinicopathologic study of 65 cases. Cancer 1984; 53:86-95.

9. Yalon D., Sam J. The presence of unilateral tonsillar enlargement in patients diagnosed with palatin tonsil lymphoma: experience at a tertiary care pediatric hospital. International Journal of Pediatric Otorhinolaryngologie 2008; 72: 9-12; 10. Ezzat A., Ibrahim E.M., El Weshi A. Localized non-Hodgkin's Iymphoma of Waldeyer's ring: clinical features, management, and prognosis of 130 adult patients. Head \& Neck 2001; 23: 547-558.

11. Bouayed K., Bousfiha A. Une tumefaction amygdalienne unilatérale de l'enfant : savoir évoquer un lymphome. Archives de pédiatrie 2006; 13 : 1460-1462.
12. King A.D., Lei K.I.K., Ahuja A.T. MRI of primary non Hodgkin's lymphoma of the palatine tonsil. Br J Radiol. 2001; 74: 226-229.

13. Hanna E., Wanamaker J., Adelstein D. Extranodal lymphomas of the head and neck. A 20-year experience. Arch Otolaryngol Head Neck Surg 1997; 123 : 1318-1323.

14. Harabuchi Y., Tsubota H., Ohguro S. Prognostic factors and treatment outcome in non-Hodgkin's lymphoma of Waldeyer's ring. Acta oncol 1997; 36: 413420.

15. Oguchi M., Likeda H., Isobe K. Tumor bulk as a prognostic factor for the management of localized aggressive non-Hodgkin's ymphoma. A survey of the japan lymphoma radiation therapy group. Int J Radiat Oncol Biol Phys 2001; 1:161-168

16. Shima N., Kobashi Y., Tsutsui K. et al. Extranodal non-Hodgkin's lymphoma of the head and neck. A clinico-pathologic study in the Kyoto-Nara area of Japan. Cancer 1990; 66:1190-7

17. Fisher R.I., Gaynor E.R., Dahlberg S., et al. Comparison of a standard regimen (CHOP) with three intensive chemotherapy regimens for advanced nonHodgkin's Lymphoma. N EngJ Med 1993; 328:1002-6

18. Gurkaynak M., Cengiz M., Ayurek S. Waldeyer's ring lymphomas. Treatment results and prognostic factors. Am J Clin Oncol 2003; 26: 437-440.

19. Aviles A., Delgado S., Ruiz H. Treatment of non-Hodgkin's lymphoma of Waldeyer's ring. Radiotherapy versus chemotherapy versus combined therapy. Eur J Cancer Oral Oncol 1996; 2:19-23. 